\title{
Reporting Items for Patent Landscapes (RIPL): The RIPL Statement
}

James A. Smith ${ }^{1,2 *}$, Zeeshaan Arshad ${ }^{2,3 *}$, Anthony Trippe ${ }^{4}$, Gary S. Collins ${ }^{1}$, David A. Brindley ${ }^{2,5-8^{* *}}$ Andrew J. Carr ${ }^{1 * *}$

1. Nuffield Department of Orthopaedics, Rheumatology and Musculoskeletal Sciences, University of Oxford, Old Road, Oxford, OX3 7LD, UK.

2. The Oxford - UCL Centre for the Advancement of Sustainable Medical Innovation (CASMI), The University of Oxford, New Richards Building, Old Road Campus, Oxford, OX3 7LG, UK

3. School of Medicine, University of Cambridge, Hills Rd, Cambridge CB2 OSP

4. Patinformatics, LLC, 565 Metro Place S. Ste. 3033 Dublin, OH, 43017, USA

5. Department of Paediatrics, Oxford University Level 2, Children's Hospital, John Radcliffe Headington, Oxford, OX3 9DU

6. Centre for Behavioural Medicine, UCL School of Pharmacy, University College London, BMA House, Tavistock Square, London WC1H 9JP, UK.

7. Harvard Stem Cell Institute, Cambridge, MA, 02138, USA.

8. USCF-Stanford Center of Excellence in Regulatory Science and Innovation (CERSI), USA

*Equal contributions

**Joint senior author

\begin{abstract}
Given evidence that the reporting quality of patent landscapes is inadequate, we have developed a reporting checklist: the Reporting Items for Patent Landscapes (RIPL) statement, to improve reporting quality. The guideline was developed through a modified Delphi methodology including input from international experts in patent landscaping.
\end{abstract}

\section{Introduction}

Patent landscapes collate and analyse information from patent publications for a variety of purposes, such as measuring technological progress, identifying innovation gaps, or studying patenting practice. They are a resource increasingly available to researchers and decision makers in the life sciences (Figure 1). Many patent landscapes gain widespread attention through publication in high impact journals viewed by key stakeholders including academics, government officials and industrial leaders. Despite their potential importance, recent evidence shows that the quality of reporting in patent landscape articles is generally insufficient (Box 1$),{ }^{1}$ potentially leading to an inability to critically appraise, interpret, and synthesise findings. Other studies have called for greater harmonisation in standards of disclosure ${ }^{2}$ and establishment of consistent practices and reporting criteria ${ }^{3}$.

Box 1: Summary of key evidence highlighting the need for reporting guidelines for patent landscapes A systematic review of patent landscape articles was previously published which presented empirical evidence of poor reporting in patent landscape articles. ${ }^{1}$ The key findings were as follows:

- Eighty-one full text articles incorporating patent landscapes were assessed for compliance against a checklist of 20 items considered by the authors to be key issues that should be reported in a patent landscape article. 
- No articles complied with all criteria and mean compliance to the checklist was $64 \%$. Just eight articles $(9.9 \%)$ reported all methodological items that would enable replication and validation of the study.

- Reporting quality did not correlate with SCImago journal ranking and there was no effect of journal type (science or other). Primary research articles were reported marginally but significantly better than other article types, such as review articles.

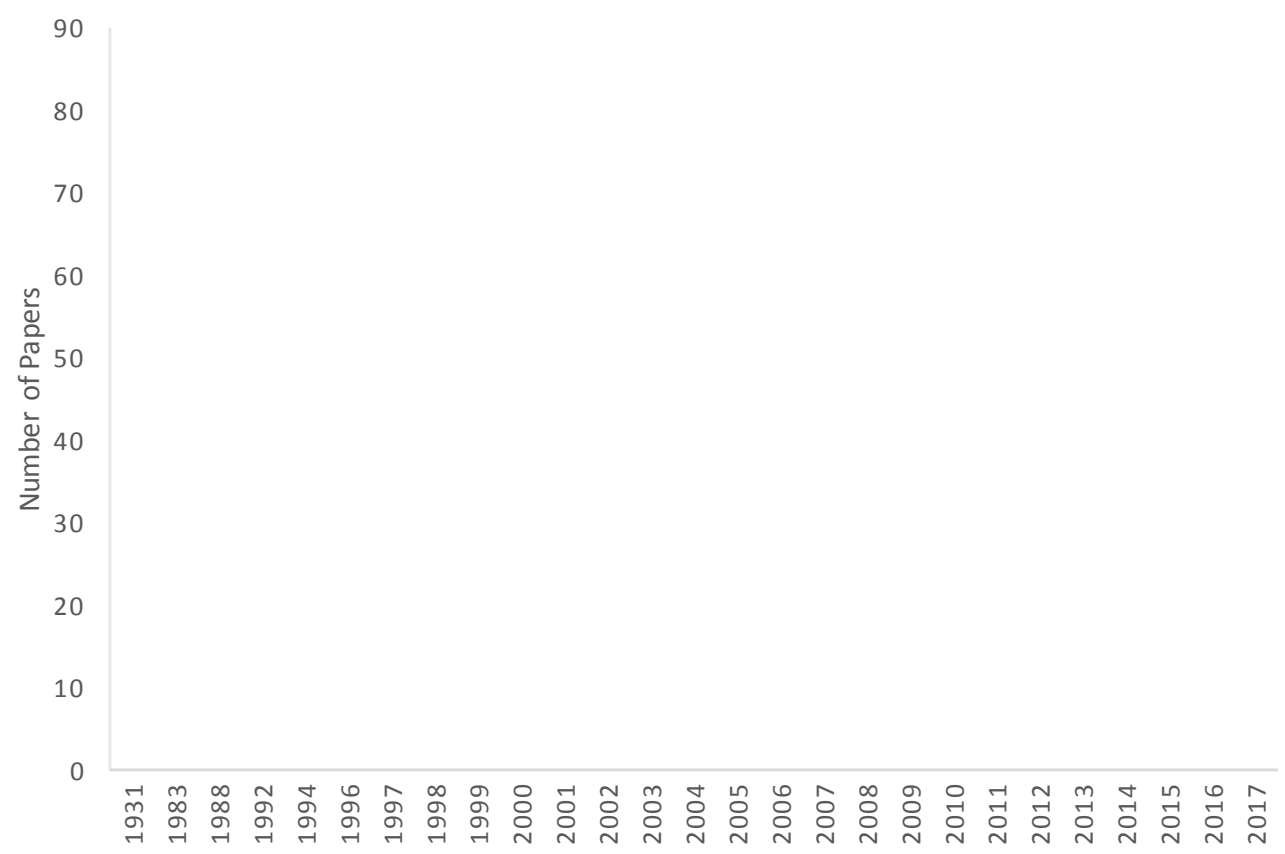

Figure 1: Number of publications mentioning the term "Patent Landscape" or "Intellectual Property Landscape" in the title, abstract or key words. Search conducted in Scopus with no start date restriction and ending on $31^{\text {st }}$ Dec 2017. Search conducted $5^{\text {th }}$ Jan 2018. Search terms: TITLE-ABSKEY (("Intellectual Property" OR "Patent") AND ("Landscape")).

Inadequate reporting has been widely reported in publications of health and biomedical research $\mathrm{h}^{4-6}$, including economic assessments ${ }^{7}$ and preclinical research ${ }^{8}$, and a large number of reporting guidelines have been developed to improve reporting quality therein (e.g. ${ }^{9-12}$ ). Guidelines have also been developed for specific research methodology reporting, including microarrays ${ }^{13}$ and data analysis in metabolomics, ${ }^{14}$ though such guidelines are much less prevalent. These and other guidelines can be found in the EQUATOR Network library of reporting guidelines (www.equator-network.org), where the development of this guideline was also registered.

Evidence suggests that the introduction of reporting guidelines can improve reporting quality in health research. For example, a systematic review of the impact of the Consolidated Standards for Reporting Trials (CONSORT) statement concluded that journal adoption of the statement is associated with improved reporting of randomised controlled trials ${ }^{15}$. A Cochrane review of the same subject similarly concluded that journal endorsement of the CONSORT statement may improve completeness of reporting ${ }^{16}$. 
To address the deficiencies in reporting quality of patent landscapes, we have developed a reporting guideline detailing minimal information that should be included in patent landscaping articles: Reporting Items for Patent Landscapes (RIPL). The guideline was developed using a modified Delphi protocol with input from international experts in patent landscaping.

\section{Checklist Development}

A modified Delphi study was conducted in late 2016 and early 2017, resulting in 48 items to be included in a consolidated reporting guideline for patent landscapes (Figure 2) (full methods are provided in supplementary material). We did not, a priori, limit the number of rounds of the Delphi study; rather, it continued until consensus was achieved on the majority of items or until responses did not change substantially between rounds. This occurred after two rounds. 

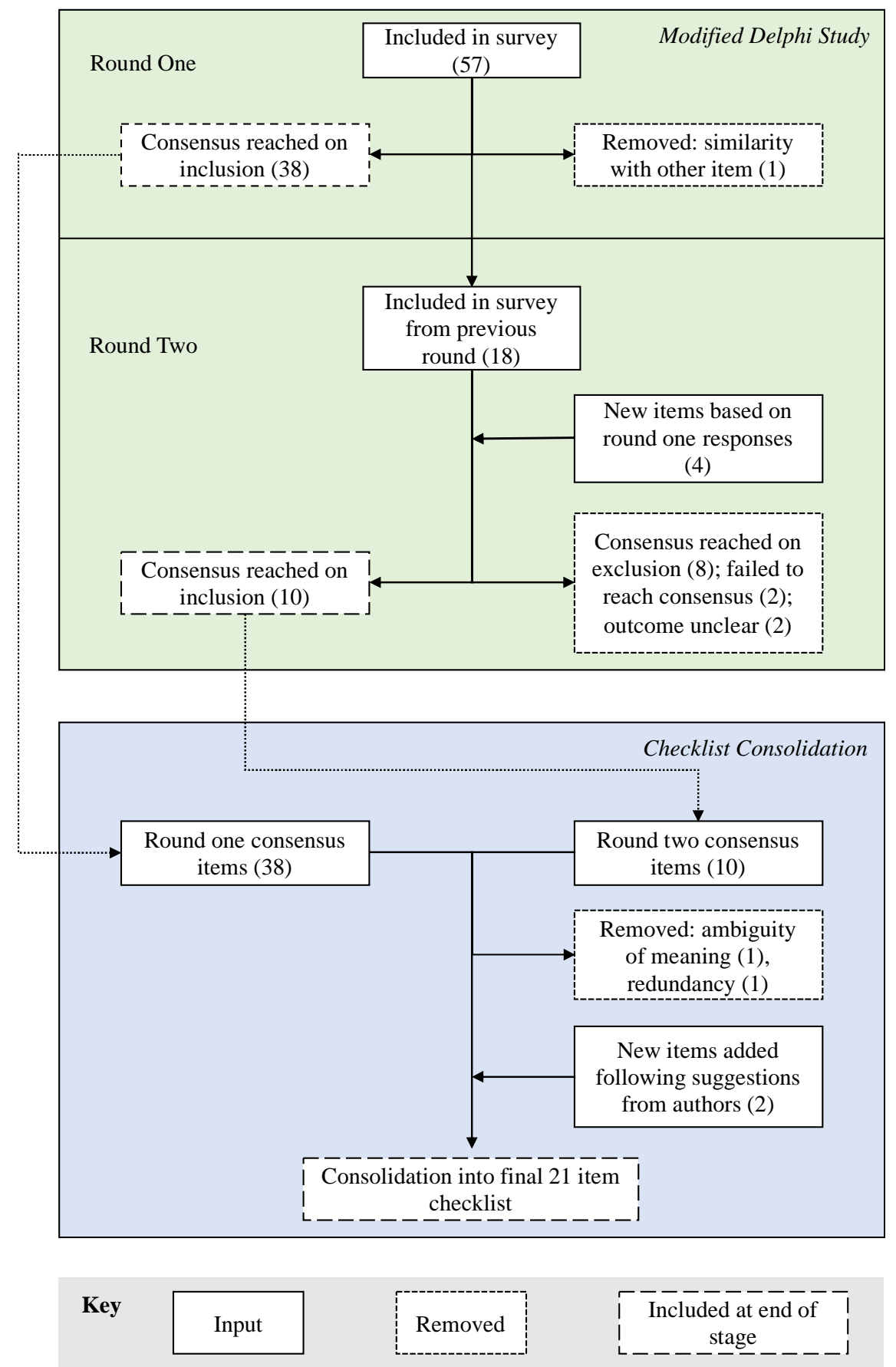

Figure 2: RIPL Checklist Development Flow Diagram. The checklist development was split into two main stages: a two-round modified Delphi study which aimed to achieve consensus on a number of proposed checklist items; and a subsequent stage where those items on which consensus was achieved were consolidated into a practical checklist. Number of items is given in brackets.

\section{Questionnaire and Participants}

A provisional questionnaire was developed based on findings and observations of our systematic review $^{1}$, discussion with relevant experts, and consultation of existing guidelines, in particular the PRISMA statement. ${ }^{10}$ This questionnaire was used in the first round of a modified Delphi study, with all items presented on a ten-point Likert scale. Twenty participants agreed to participate in the first round of the Delphi study, representing a participation rate of $29 \%$. The participants were authors of 
academic patent landscape articles, involved in the production of patent landscapes in a commercial setting, or had previously been involved in the development of guidelines for reporting of research. Of the remaining experts who were contacted, 11 declined to participate and 39 failed to respond. One respondent agreed to participate in the study but did not complete the first round, and another completed the first round but did not complete the second round (Figure 3). Participants were of a range of geographical locations, backgrounds and ages (Supplementary Figure 1) and both male and female.

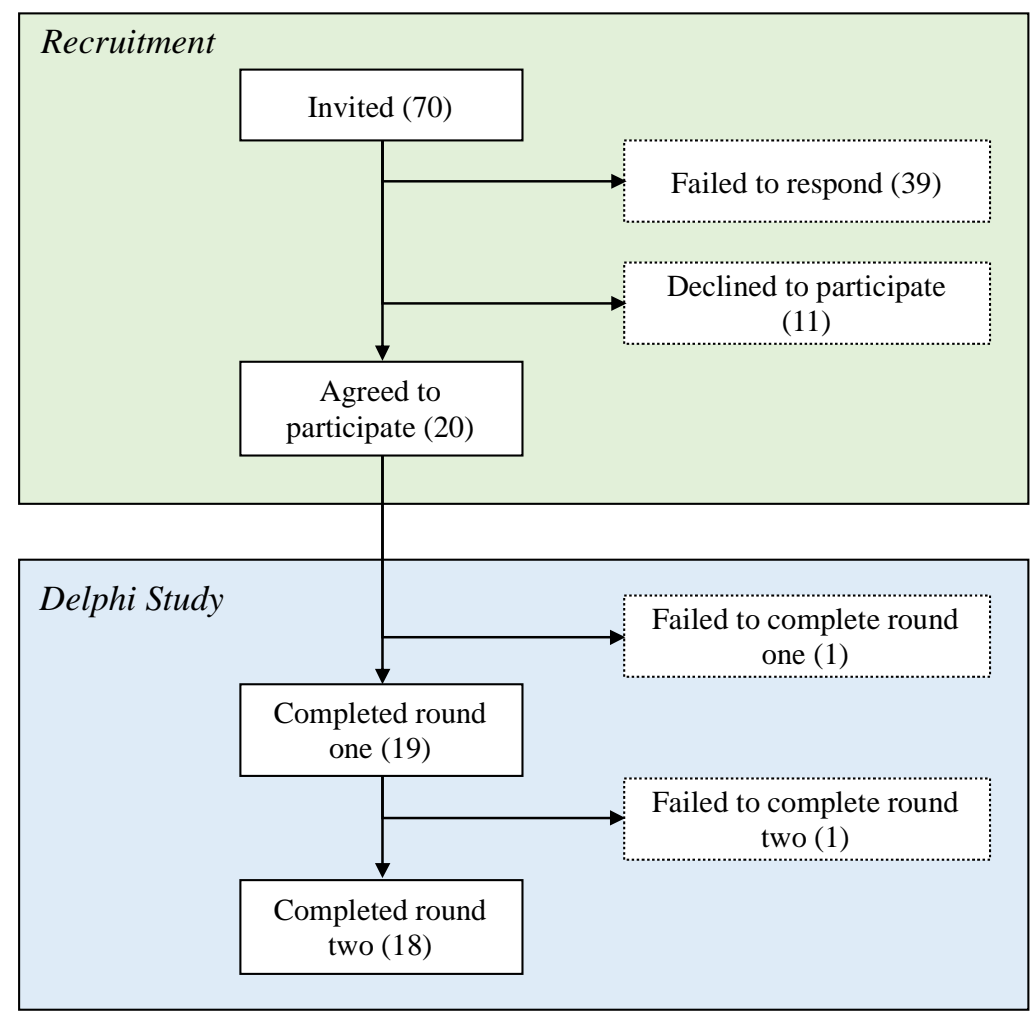

Figure 3: Participant recruitment and participation. Number of experts is given in brackets.

\section{Round One}

The initial survey round represented an over inclusive list of 57 candidate reporting items that could theoretically be reported in a patent landscaping study (Supplementary material 4). These were agreed upon through searches of the extant literature, including the PRISMA checklist ${ }^{10}$, results of our previous systematic review ${ }^{1}$, and discussion amongst the authors. In round one, consensus was reached on the inclusion of 38 items so these items were not included in the subsequent round, and one item which was removed due to similarity in meaning to another item. No items were excluded. Consensus was not reached on 18 candidate items, and these were therefore included in the second round.

\section{Round Two}

The second round included the 18 candidate items on which consensus was not reached in the first round, in addition to four new candidate items added as a result of qualitative responses provided by survey participants during round one, leading to a total of 22 candidate items. The group mean, 
standard deviation, median, and range of scores for each item also present in round one were reported back to respondents. Consensus was reached on the inclusion of ten items and the exclusion of eight items. For two items, consensus was reached but the inclusion or exclusion criteria were not met, and thus the outcome was unclear. For two further items, consensus could not be achieved but responses were stable.

In addition, in the second round of the study, we proposed a definition of a patent landscape, on which consensus and agreement was reached.

\section{Consolidation of Consensus Items}

At the end of round two, consensus had been reached on the inclusion of 48 items in the reporting guideline. Because having a large number of reporting items in a checklist is likely to reduce its practicality and usability, and because reporting guidelines include a median of 21 checklist items ${ }^{17}$ the authors consolidated the consensus items into a 20 item final checklist, grouping related items into single items. 46 of the 48 items on which consensus was reached are represented in the final checklist; two items were removed due to ambiguity of meaning. Two additional items were added in the consolidation phase following suggestion by co-authors, resulting in a final 21 item checklist. The final checklist was checked for comprehension and unambiguity by all authors and revised where required.

\section{Definition of a Patent Landscape}

Consensus was achieved on the definition of a patent landscape to be the following (adapted from ref: $^{3}$ and previously published in ref: ${ }^{1}$ ): "A landscape is an analysis of the relationships between multiple sets of indicators or of those indicators measured against temporal, technical or spatial dimensions. At least one of the indicators, in the case of a patent landscape, are patent publications or some aspect thereof. A landscape seeks to encompass an entire population of relevant data, rather than a random sample drawn from that population".

\section{Final Checklist}

The final checklist of recommended reporting items is included in Table 1. During the modified Delphi study, it was pointed out by some participants that, although we listed each item within a certain section (e.g. Abstract, Introduction, etc.), in some cases it may be more appropriate to include information in supplementary material (e.g. item no. 15, list of patent numbers). Some participants also noted that patent landscapes are not always written as structured research papers. We therefore clarified at the beginning of round two that the sections are provided for the purpose of providing structure to the reporting guideline, and that in practice most items could be included in any part of the paper, or alternatively in the supplementary material.

Table 1: Checklist of items to be reported in a patent landscaping article

Item No./ Topic Checklist Item
Section

Title 


\begin{tabular}{|c|c|c|}
\hline 1 & Title & $\begin{array}{l}\text { Identify that the article includes a patent landscape and state the } \\
\text { subject matter under investigation (e.g. gene editing technologies) }\end{array}$ \\
\hline \multicolumn{3}{|c|}{ Summary/Abstract } \\
\hline 2 & $\begin{array}{l}\text { Background and } \\
\text { summary }\end{array}$ & $\begin{array}{l}\text { Provide a summary which includes the background, rationale, } \\
\text { results and main findings in the context of the aims }\end{array}$ \\
\hline \multicolumn{3}{|c|}{ Introduction } \\
\hline 3 & Rationale & $\begin{array}{l}\text { Describe the rationale for the study, including relevant background } \\
\text { information and the potential impact of the investigation }\end{array}$ \\
\hline 4 & Aims & Describe the aims of the study \\
\hline \multicolumn{3}{|c|}{ Methods } \\
\hline 5 & Search & $\begin{array}{l}\text { State the databases and patent offices searched, the dates on } \\
\text { which the searches were conducted, and the components of the } \\
\text { patents searched. Include the search terms used for all databases } \\
\text { searched. }\end{array}$ \\
\hline 6 & $\begin{array}{l}\text { Selection } \\
\text { criteria }\end{array}$ & $\begin{array}{l}\text { Include details of the selection criteria of patents to be included in } \\
\text { the patent landscape, including the subject matter of those patents }\end{array}$ \\
\hline 7 & $\begin{array}{l}\text { Identification of } \\
\text { relevant } \\
\text { patents }\end{array}$ & $\begin{array}{l}\text { If applicable, state how patents identified in searches were sorted } \\
\text { for relevance }\end{array}$ \\
\hline 8 & Data extraction & $\begin{array}{l}\text { List and define all information that was collected from the patent } \\
\text { documents in the patent landscape (e.g. technical area, date of } \\
\text { publication), if any software was used to extract the data, and the } \\
\text { protocol if the information sought from a patent document was not } \\
\text { available }\end{array}$ \\
\hline 9 & Analysis & Describe any analysis and synthesis of results \\
\hline 10 & $\begin{array}{l}\text { Patent family } \\
\text { designation }\end{array}$ & $\begin{array}{l}\text { State the source of patent family designations (e.g. Derwent or } \\
\text { INPADOC) if any analysis incorporated patent families }\end{array}$ \\
\hline \multicolumn{3}{|c|}{ Results } \\
\hline 11 & Patent selection & $\begin{array}{l}\text { State the number of patents (or patent families) assessed for } \\
\text { eligibility, the number included in the study, and the reasons for } \\
\text { exclusion at each stage of the process. A flow diagram may be } \\
\text { useful }\end{array}$ \\
\hline 12 & $\begin{array}{l}\text { Data } \\
\text { standardisation }\end{array}$ & $\begin{array}{l}\text { Provide details of any steps taken to standardise or normalise the } \\
\text { data. Examples would typically include correcting misspellings, and } \\
\text { discussion of assumptions associated with licensing or mergers and } \\
\text { acquisitions. }\end{array}$ \\
\hline 13 & Summary & $\begin{array}{l}\text { Summarise the patents included in the study (e.g. with reference to } \\
\text { the data extracted from them, geographical distribution, temporal } \\
\text { distribution) }\end{array}$ \\
\hline 14 & Analysis & $\begin{array}{l}\text { Present and explain the results of any analysis (statistical or } \\
\text { otherwise) conducted. Include details of settings used for any } \\
\text { analyses (e.g. spatial concept maps). For any temporal analysis, } \\
\text { include details of what year convention was used (e.g. earliest } \\
\text { priority year, application year, publication year) }\end{array}$ \\
\hline
\end{tabular}



for this)

\begin{tabular}{|c|c|c|}
\hline \multicolumn{3}{|c|}{ Discussion } \\
\hline 16 & Summary & $\begin{array}{l}\text { Summarise the main findings, how they relate to the aims, and to } \\
\text { whom they may be relevant }\end{array}$ \\
\hline 17 & Limitations & $\begin{array}{l}\text { Discuss any limitations of the work in the context of the reliability } \\
\text { of the conclusions; include discussion of limitations related to the } \\
\text { methodology and software. If applicable, include information } \\
\text { relating to how sources of error were reduced }\end{array}$ \\
\hline 18 & Context & $\begin{array}{l}\text { Explain how the findings relate to other studies in the field, how the } \\
\text { study builds upon previous work, its potential impact, and } \\
\text { implications for future research }\end{array}$ \\
\hline 19 & Conclusions & $\begin{array}{l}\text { Provide a conclusion which gives a general interpretation of the } \\
\text { results in the context of other evidence }\end{array}$ \\
\hline \multicolumn{3}{|c|}{ Other } \\
\hline 20 & $\begin{array}{l}\text { Conflict of } \\
\text { Interest }\end{array}$ & Disclose any potential conflicts of interest \\
\hline 21 & Funding & $\begin{array}{l}\text { Disclose any sources of funding for the study and the role of the } \\
\text { funder in the study, and any other support received during the } \\
\text { study (e.g. supply of data) }\end{array}$ \\
\hline
\end{tabular}

\section{Discussion}

Patent landscapes have been published focussing on a broad range of topics, including inhalers ${ }^{18}$, RNA interference ${ }^{19}$, antibody therapeutics ${ }^{20}$, transgenic cotton ${ }^{21}$, and many others. They have been used in attempts to identify innovation drivers ${ }^{22}$, predict product development ${ }^{23}$, analyse patent practices $^{24}$ and assess global technological progress. Clearly, therefore, their findings could underpin important decision-making and analysis by academics, industry and government. Despite this, there is evidence that the quality of reporting in patent landscape articles in the life sciences is insufficient ${ }^{1}$

We have previously noted that the process of conducting a systematic review is analogous to that of conducting a patent landscape. However, in many cases, patent landscape articles are published as article types other than 'original research' and may avoid the close methodological scrutiny that would accompany the publication of a systematic review. For systematic reviews, and many other areas of health research, reporting checklists, flowcharts, or other guidance documents have been developed to improve reporting quality, and there is evidence suggesting that they have done so ${ }^{15,25,26}$.

The vast majority of reporting guidelines developed to date focus on health research reporting. Outside of specifically health research, however, the development and implementation of reporting guidelines has been much more limited. It is plausible that the reason for this is related to the direct impact on physician decision-making elicited by published health research, which in turn directly impacts patient care. For example, the findings of a systematic review comparing the efficacy of two 
interventions may directly influence the choice of therapeutic prescribed by a physician. Therefore, the ability of both the physician and the publisher of the research to evaluate the methodology, results, and other aspects of the paper is of paramount importance, and reporting guidelines to facilitate this have resulted.

Patent landscapes, by contrast, are more influential in the innovation process, and may thus have considerable weight in determining technology development. Largely, decision-making based on patent landscaping will occur at the system-level (such as in strategic decisions in government or companies) and therefore have broad reaching impact. Given, for example, the UK government's investment in a number of patent landscape reports which, among other things aim to help people "to consider the direction of future funding" 27 , it is apparent that patent landscapes are expected to be relied on for such purposes. At the other end of the spectrum, individual researchers or research groups may rely on the findings of patent landscape articles to determine research focus (e.g. if a patent landscape indicates that there are challenges with freedom-to-operate in an area, that area may be avoided by researchers). In both cases the eventual impact on society could be significant, and therefore the requirement for evaluation, synthesis and reproduction of such work is significant. For these reasons, we have developed a reporting checklist: RIPL.

\section{Scope}

The RIPL checklist is intended for patent landscape articles, as defined above, that are published in academic journals. The literature that provided the justification for this checklist development focussed on the life sciences; however, the checklist is not specific to the life sciences and can be applied uniformly regardless of the discipline. In particular, authors and reviewers of patent landscape articles, as well as journal editors who publish them, could benefit from its use. For authors, consulting the checklist prior to and during the development of a manuscript will help to ensure that it is well reported, facilitating fair evaluation of the manuscript by peer reviewers, reproducibility, and improving the feasibility of evidence synthesis. For reviewers and journal editors, the checklist provides a straightforward means to assess compliance to a consensus-based set of minimal criteria that should be reported in a patent landscape article. Journal endorsement of these guidelines could improve the quality of patent landscape articles accepted for publication.

While this checklist is intended primarily for use in academic research, reporting the recommended items in any patent landscape may improve the quality of the report and facilitate its assessment by others. Analysts or researchers in industry, government, or other organisations producing patent landscape reports for internal or external publication may therefore benefit from following this reporting checklist.

It should be stressed that this guidance does not extend to recommendations regarding methodologies; rather, it focusses specifically on reporting. For information on conducting patent landscapes, useful resources are available from the World Intellectual Property Office (WIPO) ${ }^{28,29}$. In particular, when planning a patent landscape, we recommend consulting, in conjunction with this paper, the "Guidelines for Preparing Patent Landscape Reports"28, which provides more granular methodological considerations for conducting patent landscapes, in comparison to this paper which provides recommended items for reporting. 


\section{Limitations}

Numerous methods have been used to develop reporting guidelines, though it is generally agreed that a consensus process should be incorporated as a central element of the methodology ${ }^{17}$. The only guidance available for development of reporting guidelines assumes that a face-to-face meeting is conducted $^{30}$; however, nearly $50 \%$ of guideline development processes do not include one ${ }^{17}$. Given the desire to include international participants in our consensus process, and the extensive resource required to conduct face-to-face meetings, we judged that a Delphi study, followed by finalisation of the checklist by the authors, represented the most reasonable methodology. It could be argued that some opportunity for discussion was lost because of this; using a Delphi study as the method for achieving consensus, however, allows anonymity and confidentiality to be maintained, reducing known negative characteristics of group dynamics such as the influence of dominant individuals ${ }^{31}$.

The outcome of a modified Delphi study, and any other consensus methodology, is influenced to some degree by the quality and expertise of the participants. We identified experts through a number of methods, some of which could introduce bias to the study (e.g. authors recommending participants for the study). Though such sampling is not ideal, it is common practice in the development of reporting guidelines and in this case it was felt necessary to ensure a sufficient sample size was recruited. To mitigate any bias that may have been introduced in this way, we welcome further comment and feedback on the reporting checklist to the corresponding authors. As practices evolve over time, or indeed become better understood, so too may the requirements for reporting. Updates to this checklist may therefore be conducted in the light of feedback received from the broad readership of this paper.

\section{Endorsement}

Journals publishing patent landscape articles may benefit from endorsing and encouraging adherence to the proposed checklist for reasons already outlined. A uniform endorsement policy across journals may help to ensure optimal uptake and ensure that the requirements for authors are the same across multiple outlets, thus reducing the burden of compliance. We therefore propose that journals adopt the following text in endorsing this guideline, for example in the 'Instructions for Authors' (adapted from the PRISMA-P 2015 statement ${ }^{32}$ ):

[This journal/organisation] endorses reporting according to the Reporting Items for Patent Landscapes (RIPL) checklist. We recommend that, while preparing an article incorporating a patent landscape, all items in the RIPL checklist that are applicable to your study are reported. Ensuring that these minimal items are reported will improve the manuscript and potentially improve its chances of eventual acceptance.'

\section{Conclusion}

Reporting checklists have been widely developed and deployed in health research, though outside of specifically health research are far less prevalent. Patents are a rich source of information that can be analysed for a variety of purposes via patent landscapes, and the resulting publications may be relied on for significant decision making. The checklist presented aims to improve the quality of reporting in such publications, but also highlights the importance of reporting quality beyond those areas in which it has traditionally been a focus. Through the implementation and continuous improvement of these 
recommendations, all stakeholders associated with patent landscaping, from authors to reviewers and editors to innovators, could benefit.

\section{References}

1. Smith, J. A., Arshad, Z., Thomas, H., Carr, A. J. \& Brindley, D. A. Evidence of insufficient quality of reporting in patent landscapes in the life sciences. Nat. Biotechnol. 35, 210-214 (2017).

2. Grant, E., Van den Hof, M. \& Gold, E. R. Patent landscape analysis: A methodology in need of harmonized standards of disclosure. World Pat. Inf. 39, 3-10 (2014).

3. Bubela, T. et al. Patent landscaping for life sciences innovation: toward consistent and transparent practices. Nat. Biotechnol. 31, 202-206 (2013).

4. Chan, A.-W. \& Altman, D. G. Epidemiology and reporting of randomised trials published in PubMed journals. Lancet Lond. Engl. 365, 1159-1162 (2005).

5. Moher, D., Tetzlaff, J., Tricco, A. C., Sampson, M. \& Altman, D. G. Epidemiology and Reporting Characteristics of Systematic Reviews. PLOS Med. 4, e78 (2007).

6. Glasziou, P., Meats, E., Heneghan, C. \& Shepperd, S. What is missing from descriptions of treatment in trials and reviews? BMJ 336, 1472-1474 (2008).

7. Neumann, P. J., Stone, P. W., Chapman, R. H., Sandberg, E. A. \& Bell, C. M. The quality of reporting in published cost-utility analyses, 1976-1997. Ann. Intern. Med. 132, 964-972 (2000).

8. Kilkenny, C. et al. Survey of the Quality of Experimental Design, Statistical Analysis and Reporting of Research Using Animals. PLOS ONE 4, e7824 (2009).

9. Kilkenny, C., Browne, W. J., Cuthill, I. C., Emerson, M. \& Altman, D. G. Improving Bioscience Research Reporting: The ARRIVE Guidelines for Reporting Animal Research. PLOS Biol. 8, e1000412 (2010).

10. Moher, D. Preferred Reporting Items for Systematic Reviews and Meta-Analyses: The PRISMA Statement. Ann. Intern. Med. 151, 264 (2009).

11. Schulz, K. F., Altman, D. G. \& Moher, D. CONSORT 2010 Statement: updated guidelines for reporting parallel group randomised trials. BMJ 340, c332 (2010).

12. Husereau, D. et al. Consolidated Health Economic Evaluation Reporting Standards (CHEERS) Explanation and Elaboration: A Report of the ISPOR Health Economic Evaluation Publication Guidelines Good Reporting Practices Task Force. Value Health 16, 231-250 (2013).

13. Brazma, A. et al. Minimum information about a microarray experiment (MIAME)-toward standards for microarray data. Nat. Genet. 29, 365-371 (2001).

14. Goodacre, R. et al. Proposed minimum reporting standards for data analysis in metabolomics. Metabolomics 3, 231-241 (2007).

15. Plint, A. C. et al. Does the CONSORT checklist improve the quality of reports of randomised controlled trials? A systematic review. Med. J. Aust. 185, (2006).

16. Turner, L. et al. Consolidated standards of reporting trials (CONSORT) and the completeness of reporting of randomised controlled trials (RCTs) published in medical journals. Cochrane Database Syst. Rev. 11, MR000030 (2012).

17. Moher, D. et al. Describing reporting guidelines for health research: a systematic review. J. Clin. Epidemiol. 64, 718-742 (2011).

18. Bowman, P. A. \& Greenleaf, D. Non-CFC metered dose inhalers: the patent landscape. Int. J. Pharm. 186, 91-94 (1999).

19. Lundin, P. Is silence still golden? Mapping the RNAi patent landscape. Nat. Biotechnol. 29, 493-497 (2011). 
20. Petering, J., McManamny, P. \& Honeyman, J. Antibody therapeutics - the evolving patent landscape. New Biotechnol. 28, 538-544 (2011).

21. Kalpana Sastry, S., Rashmi, H. B. \& Badri, J. Research and development perspectives of transgenic cotton: Evidence from patent landscape studies. J. Intellect. Prop. Rights 16, 139-153 (2011).

22. Agarwal, P. \& Searls, D. B. Can literature analysis identify innovation drivers in drug discovery? Nat. Rev. Drug Discov. 8, 865 (2009).

23. Korting, H. C., Blecher, P., Schäfer-Korting, M. \& Wendel, A. Topical liposome drugs to come: what the patent literature tells us. A review. J. Am. Acad. Dermatol. 25, 1068-1071 (1991).

24. Sternitzke, C. An exploratory analysis of patent fencing in pharmaceuticals: The case of PDE5 inhibitors. Res. Policy 42, 542-551 (2013).

25. Smidt, N. et al. The quality of diagnostic accuracy studies since the STARD statement: has it improved? Neurology 67, 792-797 (2006).

26. Prady, S. L., Richmond, S. J., Morton, V. M. \& Macpherson, H. A systematic evaluation of the impact of STRICTA and CONSORT recommendations on quality of reporting for acupuncture trials. PloS One 3, e1577 (2008).

27. Eight great technologies: the patent landscapes - Publications - GOV.UK. Available at: https://www.gov.uk/government/publications/eight-great-technologies-the-patent-landscapes.

(Accessed: 21st November 2016)

28. World Intellectual Property Office. Guidelines for Preparing Patent Landscape Reports. (2015). 29. The WIPO Manual on Open Source Patent Analytics.

30. Moher, D., Schulz, K. F., Simera, I. \& Altman, D. G. Guidance for Developers of Health Research Reporting Guidelines. PLoS Med. 7, (2010).

31. Hsu, C.-C. \& Sandford, B. A. The Delphi technique: making sense of consensus. Pract. Assess. Res. Eval. 12, 1-8 (2007).

32. Moher, D. et al. Preferred reporting items for systematic review and meta-analysis protocols (PRISMA-P) 2015 statement. Syst. Rev. 4, 1 (2015).

\section{Acknowledgements}

This study is funded primarily by the UK Medical Research Council via JS' DPhil funding. The authors also acknowledge support from the CASMI Translational Stem Cell Consortium (CTSCC) and the SENS Research Foundation. We are extremely grateful for the input from the experts that participated in the Delphi study and provided valuable input during the development of this guideline. We are also grateful to the Equator Network for assistance with early work that established the need for this guidance.

\section{Disclosures}

DB is a stockholder in Translation Ventures Ltd. (Charlbury, Oxfordshire, UK), IP Asset Ventures Ltd. (Oxford, Oxfordshire, UK), and Biolacuna Ltd (Oxford, Oxfordshire, UK), companies that among other services provide biomanufacturing, regulatory, and financial advice to pharmaceutical clients. DB also is subject to the CFA Institute's codes, standards, and guidelines, so he must stress that this piece is provided for academic interest only and must not be construed in any way as an investment recommendation. Additionally, at time of publication, DB and the organizations with which he is 
affiliated may or may not have agreed and/or pending funding commitments from the organizations named herein. JS has consulted with life science focussed companies, including some involved in patent landscaping. 